\title{
Paraclinical management of SARS-CoV-2 infection in paediatric patient
}

\author{
Ana-Maria Davitoiu ${ }^{1,2}$, Sorina Chindris ${ }^{1,2}$, Mirela Silvia Iancu ${ }^{1,2}$, Iulia Tincu ${ }^{1,2}$, \\ Luminita Elena Spatariu, ${ }^{1,2}$, Doina Anca Plesca ${ }^{1,2}$ \\ ${ }^{1}$ Faculty of Medicine, "Carol Davila" University of Medicine and Pharmacy, Bucharest, Romania \\ 2"Victor Gomoiu" Children's Hospital, Bucharest, Romania
}

\begin{abstract}
The world is facing, after a hundred years, a new pandemic - infection with the SARS-CoV-2 virus. Establishing a therapeutic protocol along with paraclinical infection management is the great challenge of the moment for healthcare professionals all around the world. This paper tries to stadialise the diagnosis methods and paraclinical investigations, establishing the time correspondence between this investigations and laboratory also radiological findings. The positive diagnosis of SARS-CoV-2 infection is made by using four methods of testing: RT-PCR molecular method, serological testing by chemiluminescence and ELISA, rapid tests for the determination of IgM and IgG antibodies. Laboratory findings are not disease-specific but studies have shown that lymphopenia occurs in $83 \%$ of paediatric hospitalized patients confirmed with SARS-CoV-2 infection.

Imaging is not recommended for diagnosis or screening purposes in patients with suspected COVID-19, the predictive value of RT-PCR test is ten times higher than the results of pulmonary $C T$.
\end{abstract}

Keywords: pandemic, coronavirus, SARS-CoV-2 infection, children, RT-PCR tests, antibodies serologic tests

\section{INTRODUCTION. EPIDEMIOLOGY}

The pandemy is defined as an epidemy that affects a large number of people and spreads beyond international borders.

Throughout human history there have been numerous pandemics, infectious diseases that often have devastating consequences. The plague, the typhus, the cholera, the yellow fever, the influenza and tuberculosis are some of this infectious diseases that have affected a large number of people over time.

We are currently going through a hard period of time, humanity is facing a new pandemic, the infection with the SARS-CoV-2 virus.

Experts, scientists and epidemiologists analyse the evolution of the pandemic while economic analysts try to anticipate the economic impact of the measures taken by authorities around the world in the fight against COVID-19.

Authorities in Wuhan, the initial epicenter of the outbreak of coronavirus in China, recorded the first case of infection in December 2019. In less than a month researchers, through a concerted effort, performed genomic sequencing. Genomic mapping brings us complex information about the particularities of this new coronavirus.

We are basically facing a virus belonging to the Coronavirus family, genus Betacoronavirus. This is a positive single-stranded RNA virus that causes severe acute respiratory syndrome. The virus was named SARS-CoV-2. $80 \%$ of its genomic sequence is identical with the virus that caused the SARS epidemic in 2002, an epidemic with a high degree of contagion and potentially life-threatening because of it's clinical severity manifestations. In the case of the SARS epidemic, 8098 cases were confirmed with a fatality rate of approximately $9 \%$ (1).

The spread of SARS CoV-2 infection in entire world has been impressive. Today we are facing with more than 7.3 million diagnosed patients and with an unknown, probably huge, number of people who have 
gone through the disease undiagnosed and that have not been yet included in statistics. To date, there are over 430,000 deaths worldwide (3). In our country, the first confirmed case with SARS-CoV-2 infection was on February 26, 2020. Since then in Romania are over 22,000 confirmed cases and more than 1,400 deaths have been recorded (2).

Unfortunately, medical staff who care for patients with SARS-CoV-2 infection are often the victims of it. Thus in the medical healthcare system one from nine infected patients belongs to the medical staff (2).

\section{WHAT WE NEED TO KNOW ABOUT SARS- COV-2 INFECTION IN CHILDREN?}

Amongs people infected with SARS-CoV-2, a percentage of $1-2 \%$ is children. The disease affects all ages even newborns and infants. The age group distribution is similar in Asia, America and Europe, the same percentages are being found in paediatric population from Romania. There are deaths amongs children (3) but their number is extremely low.

\section{DIAGNOSIS MANAGEMENT OF SARS-COV-2 INFECTION IN CHILDREN}

To diagnose the COVID-19 disease, testing should be performed in order to identify the SARS-CoV-2 infection.

The National Institute of Public Health, through its update from April 28, 2020, recommends to test suspicious cases according to the case definition for acute respiratory syndrome with the new coronavirus (COVID-19) (2).

Figure 1 presents the testing methodology in accordance with the recommendations of the National Institute of Public Health.
The definition of a suspicious case includes 2 aspects - the epidemiological context and the presence of respiratory symptomatology. The epidemiological context addresses extremely well-defined aspects regarding travel history and contact with suspicious or confirmed COVID-19 people. It also analyzes the membership to social clusters. The other aspect, that of the clinical manifestations, includes people of any age that have symptoms of the triad: fever, cough, difficulty of breathing (2).

The suspicious case selected according to the case definition for the acute respiratory syndrome with the new coronavirus will be tested by the nucleic acid amplification method, real-time PCR (RT-PCR). A positive result on RT-PCR test turns the suspicious case into a confirmed case defined as a person with laboratory confirmation of SARS-CoV-2 infection regardless of the presence or absence of clinical signs and symptoms (2).

In case of the paediatric patients, they often present at the onset of the disease, respiratory symptomatology characterized by fever, cough, respiratory difficulties, the same clinical symptomatology as in adults (2).

Nevertheless a significant percentage of children may experience nonspecific digestive clinical manifestations, neurological clinical manifestations, eruptive manifestations and skin lesions (4). Thus, knowing these atypical forms of presentation at onset of the disease, helps the clinician to make the correlation between nonspecific onset of the disease (outside the definition of a suspicious case) and SARS-CoV-2 infection.

The positive diagnosis of SARS-CoV-2 virus infection is made by using four test methods: molecular RT-PCR method, serological testing by chemiluminescence and by ELISA method, rapid tests for the determination of IgM and IgG antibodies (5).

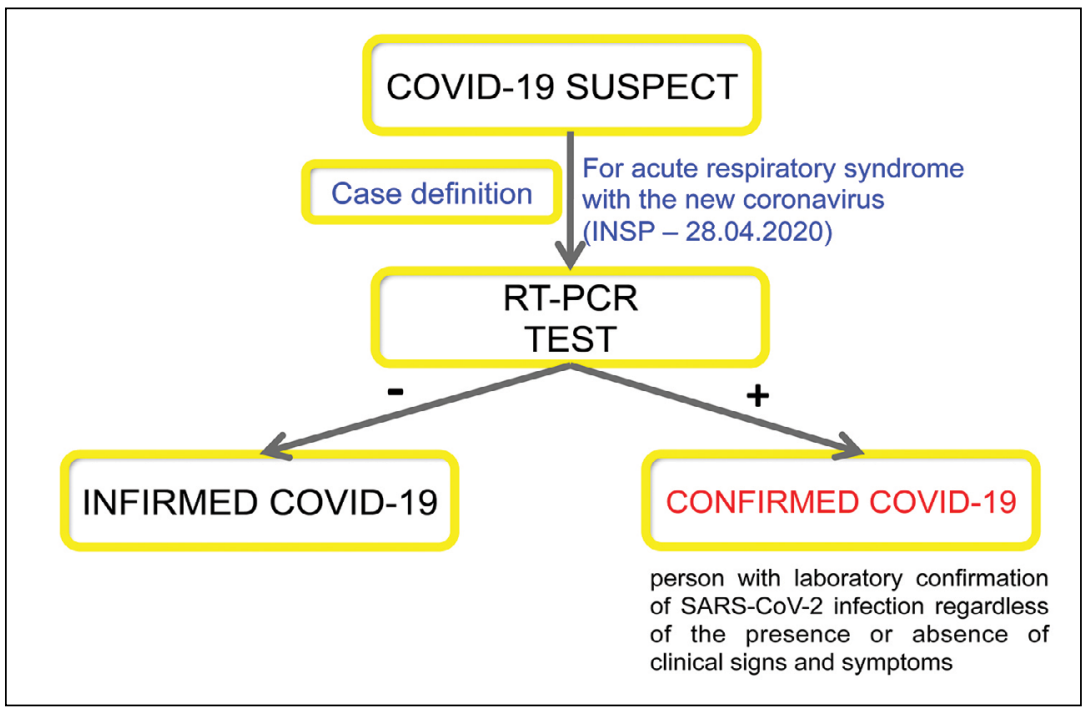

FIGURE 1. Methodology for testing the suspected cases of SARS-CoV-2 infection (2) 


\section{RT-PCR}

Reverse transcriptase polymerase chain reaction is considered the "gold standard" method for diagnosing SARS-CoV-2 virus infection. The test has a high specificity and a sensitivity up to $98 \%$ (6).

The test uses sample of biological material collected from the upper airways (nasopharyngeal / oropharyngeal exudate) or from the lower airways (sputum, tracheal aspirate, tracheobronchial lavage) in patients with severe acute respiratory syndrome (1).

Physicians working with suspected COVID-19 patients know the importance of these results in the subsequent management of the patient. The processing time of the samples by RT-PCR until the results are obtained is approximately 4 hours.

There is a risk of a false-negative reaction that is why the methodology provided by the National Institute of Public Health establishes to perform 2 tests with a distance of at least 24 hours between them.

\section{Antibodies serological testing}

The second method used to diagnose SARS-CoV-2 infection in children is antibodies serological testing. Serological testing is used as a complementary method to RT-PCR testing. Serological testing can be performed by quantitative or qualitative methods.

Rapid serological tests are insufficiently validated. These tests can be used as a complementary method to RT-PCR testing for serological screening or to check the immune response in large populations (5).

Figure 2 illustrates how to use rapid tests to identify SARS-CoV-2 infection.

A positive IgM result at serological testing places the paediatric patient as possible COVID-19 infected. This means that this patient will be evaluated clinically and treated in hospital according to the protocol. A negative IgM result at serological testing will be validated by performing RT-PCR which confirms or infirms the COVID-19 diagnosis.

Presence of IgG antibodies at serological testing shows that a person has previously been infected with the SARS-CoV-2 virus. Aat this moment there is insufficient medical evidence to support long-term immunity with $\mathrm{IgG}$ antibodies to the SARS-CoV-2 virus infection (5).

\section{The serological tests}

These tests determine the presence of IgM and IgG antibodies. Antibodies are proteins produced by the immune system to defend itself against infection. As a result, the production of these antibodies takes place over time. Thus, IgM antibodies can be detected in the blood from the day 7 after the infectious contact, while IgG antibodies appear after day 10 . The presence of these antibodies is an indicator of the development of the immune response and clinical recovery $(7,8)$. Figure 3 shows the dynamics of laboratory spe-

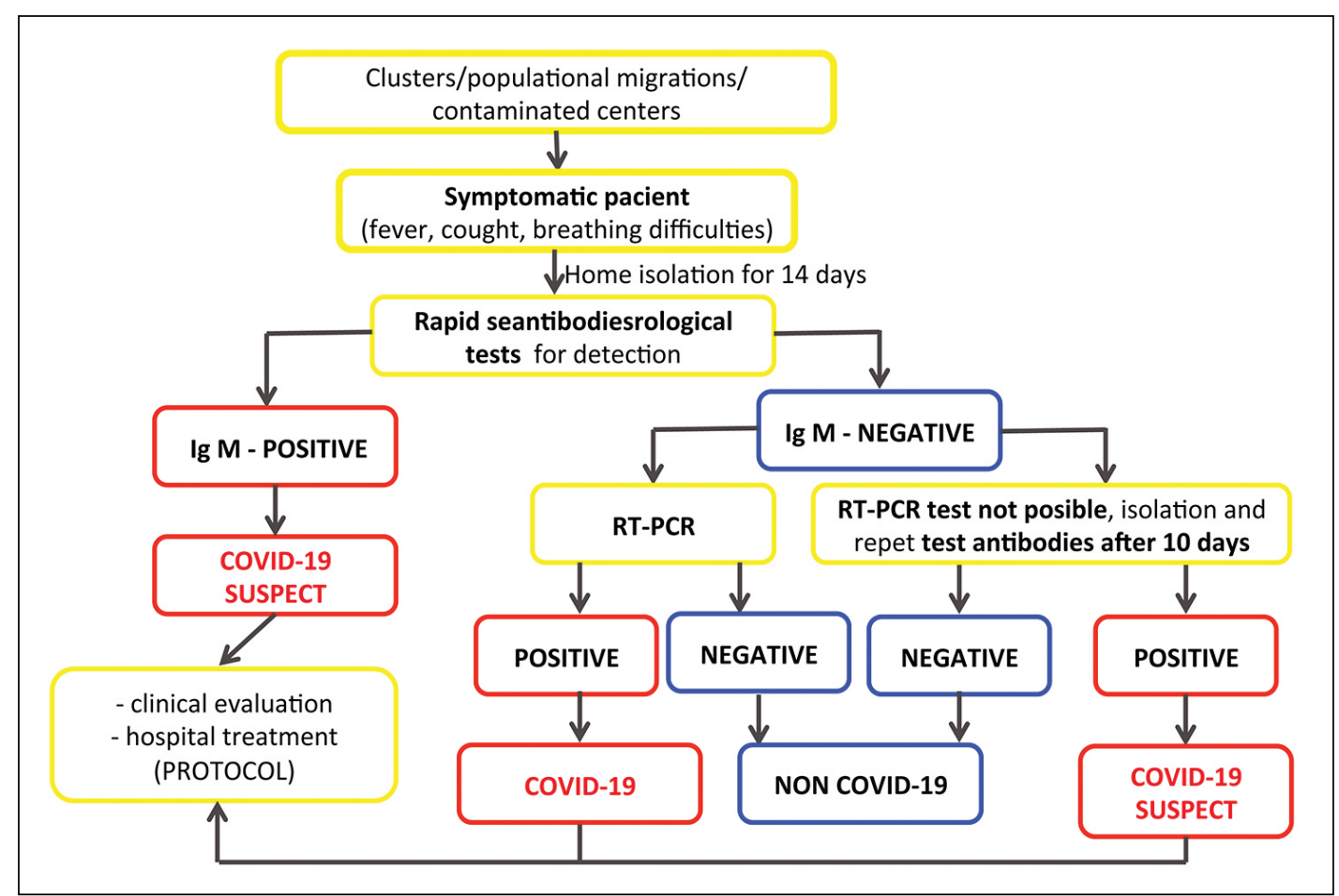

FIGURE 2. The use of rapid antibodies serological tests in clusters / population migrations / contaminated centers - adapted after (5) 


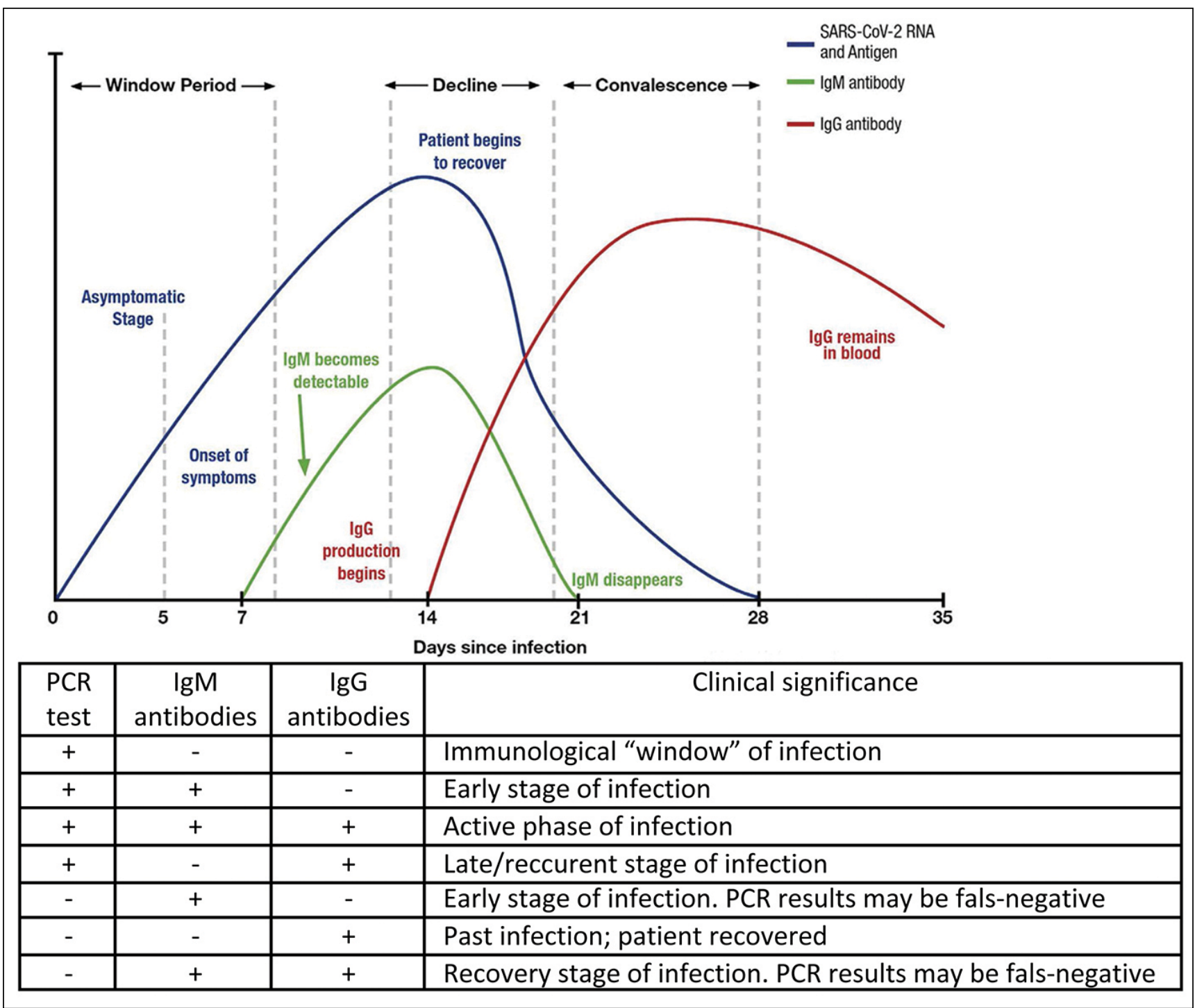

FIGURE 3. Dynamics of the laboratory specific investigations to establish the diagnosis of SARS-CoV-2 infection - adapted after (8)

cific investigations to establish infection with the new coronavirus.

The limits of this test makes it useful for research purposes and for large clusters or population migrations (5).

The serological testing is a diagnostic method that can be used to assess the prevalence of SARS-CoV-2 virus infection. The information provided by serological testing is of both medical and socio-economic importance. Thus, it can be widely used to develop strategies for relaxing the measures imposed by social distancing and to relaunch the economy (7).

\section{LABORATORY INVESTIGATIONS}

These are the investigations performed at paediatric patients that are being hospitalized with suspected SARS-CoV-2 infection until RT-PCR tests confirm or infirm the infection.
Laboratory investigations are not specific to the disease but studies show that most of the hospitalized patients, later confirmed with SARS-CoV-2 infection, have changes in blood counts, inflammatory markers (CRP, VSH), D-dimers levels, ferritin levels, procalcitonin levels, glycemia levels, transaminases and creatine phosphokinase levels, lactate dehydrogenase levels etc. $(5,9)$.

Co-infections are possible that is why paediatric patients who meet the case definition criteria should be tested for SARS-CoV-2 virus infection regardless of the presence of another respiratory pathogen.

Lymphopenia is an haematological finding associated with SARS-CoV-2 infection that occurs in approximately $83 \%$ of the paediatric hospitalised pacients (5).

Patients with severe evolution of COVID-19 disease associate lymphopenia with neutrophilia as well as with increased levels of transaminases, creatine 
phosphokinases, lactate dehydrogenases, CRPs, ferritins levels (5).

Mortality and morbidity in the paediatric population diagnosed with SARS-CoV-2 virus infection are higher in those pacients with increased levels of Ddimers and lymphopenia. Intensive care patients who have an unfavorable prognosis often have elevated procalcitonin levels while critically ill patients (with a possible underlying immune disorder) have increased levels of nonspecific inflammatory markers (5).

\section{IMAGING IN SARS-COV-2 INFECTION IN CHILDREN}

The use of information provided by imagistical evaluation in the paediatric patient infected with the SARS-CoV-2 virus remains the most controversial aspect in the current pandemic.

\section{General principles for the use of imaging in paediatric patients infected with SARS-CoV-2 virus (10)}

- imaging evaluation for diagnostic or screening purposes is not recommended in patients with suspected COVID-19 disease (predictive value of RT-PCR is ten times higher than pulmonary CT)

- imaging evaluation is not recommended in patients with suspected or mild forms of the disease

- imaging evaluation is reserved for patients with unsatisfactory evolution of respiratory status

- imaging evaluation is useful in monitoring the appearance and the evolution of the disease complications.

Imaging investigations that can be performed for diagnostic purposes in paediatric patients with SARSCoV-2 virus infection are: lung radiography, computer tomography examination, thoracic ultrasound.

If imaging is used, plan radiography is the first intention (5).

\section{Pulmonary radiography}

The pulmonary radiography shows no changes at onset and in mild forms of the disease which is why it should not be routinely recommended.

In the case of paediatric hospitalized patients diagnosed with SARS-CoV-2 virus infection, the pulmonary radiography identify radiological changes in 69-
$80 \%$ of the cases. Maximum lesions appear on day 10-12 from the onset of the disease (11). Radiologically detected lung lesions are frequently bilateral, peripheral, they often occupy the lung bases and appears like inhomogeneous lung opacities $(10,11)$.

The discrepancy between lung parenchyma damage and reduced lung effusions is noticeable (11).

If lung radiography is used, it is recommended:

- to use portable devices (at the patient's bed)

- to use personal protective equipment

- to disinfect the used equipment (there is the possibility for the virus to remain on the equipment and surfaces for up to 72 hours).

In children both pulmonary radiography and pulmonary computer tomography examination provide images of atypical or organized pnaeumonia often multilobar and/or bilateral $(10,11)$.

\section{Pulmonary computer tomography examination}

There are no specific lung changes at computer tomography examination.

CT findings at the onset of the COVID-19 disease are: pulmonary "matt glass" opacities located bilateral predominantly peripheral without nodular appearance, adenopathy or pleurisy. In evolution of the disease, as the lung damage progresses, the lung architecture changes, the opacities confluence and may appear bronchiectasis (10).

In the paediatric patient the aspects obtained by computer tomography are similar to those described above but of a smaller magnitude compared to those found in adults. In addition, with the remission of the disease (5), a complete pulmonary restitution is found.

CT examination is not used in the primary screening of the disease. Routine pulmonary CT examination of large cohorts of patients involves large financial resources (cost / maneuver and protective equipment), high risk of transmitting the disease to medical and support staff and to other patients within the radiology departments $(5,11)$.

\section{CONCLUSIONS}

Establishing the correct paraclinical management for the diagnosis of SARS-CoV-2 infection allows a rapid and accurate diagnosis for every suspected patient worldwide. The assiduous medical research made in this period tries to establish an unique therapeutic protocol in order to save lives.

Conflict of interest: none declared Financial support: none declared 


\section{REFERENCES}

1. World Health Organisation https://www.who.int/ith/diseases/sars/en SARS Severe Acute Respiratory Syndrome.

2. Institutul National de Sanatate Publica - http://www.cnscbt.ro/ Infectia cu noul coronavirus SARS-CoV-2.

3. Worldometers.com.

4. Deville JG, Song E et al. Coronavirus disease 2019 (COVID-19): Considerations in children. Uptodate. 2020.

5. Shastri DD et al. COVID 19 second bulletin. Indian Academy of Pediatrics. 2020:5-60.

6. Watson J, Whiting PF et al. Interpreting a COVID-19 test result. BMJ. 2020.

7. Zhengtu Li et al. Development and clinical application of a rapid IgM-IgG combined antibody test for SARS-CoV-2 infection diagnosis. J Med Virology. 2020.
8. SARS-CoV-2 (COVID-19): Diagnosis by IgG/lgM Rapid Test. CliniSciences. 2020. Available at https://www.clinisciences.com/it/ read/newsletter-26/sars-cov-2-covid-19-diagnosis-by-2264.html.

9. TinkuJ, Moslehi MA (Chief Editors). International pulmonologist's consensus on COVID-19. Available at https://www.unah.edu.hn/ dmsdocument/9674-consenso-internacional-de-neumologos-sobrecovid-19-version-ingles.

10. Bickle I, Bell DJ et al. COVID-19. Radiopedia. 2020.

11. Kanne JP, Chung JH et al. Essentials for Radiologists on COVID-19: An Update - Radiology Scientific Expert Panel. Radiology. 2020. 